\title{
Almost minimum diameter orientations of semicomplete multipartite and extended digraphs
}

\author{
Gregory Gutin \\ Department of Computer Science \\ Royal Holloway, University of London \\ Egham, Surrey, TW20 0EX, UK \\ gutin@cs.rhul.ac.uk \\ Khee Meng Koh \\ Department of Mathematics \\ National University of Singapore \\ 119260 Singapore \\ matkohkm@nus.edu.sg \\ Eng Guan Tay \\ Mathematics and Mathematics Education Group \\ Nanyang Technological University \\ 259756 Singapore \\ egtay@nie.edu.sg \\ Anders Yeo \\ Department of Computer Science \\ Royal Holloway, University of London \\ Egham, Surrey, TW20 0EX, UK \\ anders@cs.rhul.ac.uk
}

\begin{abstract}
An orientation of a digraph $D$ is a spanning subdigraph of $D$ obtained from $D$ by deleting exactly one arc between $x$ and $y$ for every pair $x \neq y$ of vertices such that both $x y$ and $y x$ are in $D$. Almost minimum diameter orientations of certain semicomplete multipartite and extended digraphs are considered, several generalizations of results on orientations of undirected graphs are obtained, some conjectures are posed.
\end{abstract}

\section{Introduction, terminology and notation}

An orientation of a digraph $D$ is a spanning subdigraph of $D$ obtained from $D$ by deleting exactly one arc between $x$ and $y$ for every pair $x \neq y$ of vertices such that both $x y$ and $y x$ are in $D$. In this paper, we consider almost minimum diameter orientations of certain semicomplete multipartite and extended digraphs. We prove several generalizations of results on orientations of undirected graphs obtained in $[5,12,14]$, remark that some others can be shown analogously, and pose two conjectures on the topic. 
While there is a large number of papers considering minimum diameter orientations of undirected graphs, see e.g. [3, $6,8,9,10,11,13,14]$, there is only one recent work [7] on minimum diameter orientations of digraphs, where the authors provide a motivation for studying the topic. It is worth noting that there are a few papers $[2,4,21]$ considering finite diameter orientations of mixed graphs (or, equivalently, of directed graphs), but none of these papers has dealt with minimizing the diameter of an orientation of a given digraph. We restrict our attention to special classes of digraphs since even the problem to check whether a given undirected graph has an orientation of diameter 2 is proved to be $\mathcal{N} \mathcal{P}$-complete by Chvátal and Thomassen [3] and the upper bound on the diameter of an orientation of an undirected graph obtained in [3] is far from best possible for many classes of undirected graphs.

We use the standard terminology and notation on digraphs as given in [1]. We still provide most of the necessary definitions for the convenience of the reader.

A biorientation of a digraph $D$ is a spanning subdigraph of $D$ obtained from $D$ by deleting exactly one arc between $x$ and $y$ for some pairs $x \neq y$ of vertices such that both $x y$ and $y x$ are in $D$. Clearly, an orientation of a digraph is a special case of biorientation. A digraph $D$ is symmetric if for every pair $x \neq y$ of vertices in $D$ either there is no arc between $x$ and $y$ or both $x y$ and $y x$ are in $D$. Symmetric digraphs are in natural correspondence to undirected graphs: for an undirected graph $G$, the symmetric digraph $\overleftrightarrow{G}$ is obtained from $G$ by replacing every edge $x y$ with the pair $x y, y x$ of arcs. Let $D=(V, A)$ be a digraph and let $x, y$ be a pair of vertices in $D$. If $x y \in A$, we say $x$ dominates $y$, and $y$ is dominated by $x$, and denote it by $x \rightarrow y$. If $X, Y \subset V$, then $X \rightarrow Y$ means that every vertex of $X$ dominates every vertex of $Y$. Notice that $X \rightarrow Y$ does not mean that there is no arc from $Y$ to $X$. The converse of a digraph $D$ is the digraph obtained from $D$ by replacing every arc $x y$ of $D$ by the arc $y x$.

All paths and cycles we consider in this paper are directed and simple. A path from $x$ to $y$ is an $(x, y)$-path. The distance, $\operatorname{dist}_{D}(x, y)$, from $x$ to $y$ in $D$ is the least length of an $(x, y)$-path if $y$ is reachable from $x$, and is equal to $\infty$, otherwise. We assume that $\operatorname{dist}_{D}(x, x)=0$ for every vertex $x \in V$. The diameter $\operatorname{diam}(D)$ of $D$ is the maximum distance from a vertex to another vertex of $D$. Clearly, $D$ is of finite diameter if and only if $D$ is strong. The minimum diameter of an orientation of a digraph $D$ will be denoted by $\operatorname{diam}_{\min }(D)$.

A digraph $D$ is semicomplete if there is at least one arc between any pair of distinct vertices of $D$. The $\left(s_{1}, s_{2}, \ldots, s_{n}\right)$-extension (or just extension) $D\left(s_{1}, s_{2}, \ldots, s_{n}\right)$ of a digraph $D$ with vertices labelled, say, $1,2, \ldots, n$ is obtained from $D$ by replacing every vertex $i$ by a set of $s_{i}$ independent (i.e. with no arc between them) vertices; more formally,

$$
V\left(D\left(s_{1}, s_{2}, \ldots, s_{n}\right)\right)=\left\{\left(p_{i}, i\right): 1 \leq p_{i} \leq s_{i}, 1 \leq i \leq n\right\}
$$

and $(p, i) \rightarrow(q, j)$ in $D\left(s_{1}, s_{2}, \ldots, s_{n}\right)$ if and only if $i \rightarrow j$ in $D$. A digraph $D$ is semicomplete $k$-partite, $k \geq 2$, if the vertices of $D$ can be partitioned into $k$ partite sets $V_{1}, V_{2}, \ldots, V_{k}$ 
such that every partite set is an independent set, but for every pair $x, y$ of vertices from distinct partite sets, $x y$ or $y x$ (or both) is in $D$. When $k=2$ we speak of semicomplete bipartite digraphs; when $k \geq 2$ we speak of semicomplete multipartite digraphs. Clearly, every semicomplete digraph with $n$ vertices is a semicomplete $n$-partite digraph, and an extension of a semicomplete digraph with $n$ vertices is a semicomplete $n$-partite digraph.

Semicomplete digraphs, semicomplete multipartite digraphs and some families of extended digraphs have been extensively studied in the literature (cf. [1] and the bibliography therein).

\section{Semicomplete multipartite digraphs}

Almost minimum diameter orientations of semicomplete digraphs and semicomplete bipartite digraphs have been studied in [7], where the following two theorems were proved. Note that Theorem A was proved for a larger family of digraphs, quasi-transitive digraphs; the digraph $\stackrel{\leftrightarrow}{K}_{1, n-1}$ clearly has no strongly connected orientation.

Theorem A If $D$ is a strong semicomplete digraph, then

$$
\operatorname{diam}_{\min }(D) \leq \max \{3, \operatorname{diam}(D)\}
$$

and the bound is sharp.

Theorem B If $D$ is a strong semicomplete bipartite digraph and $D \neq \overleftrightarrow{K}_{1, n-1}$, then

$$
\operatorname{diam}_{\min }(D) \leq \max \{5, \operatorname{diam}(D)\}
$$

and the bound is sharp.

Based on Theorems A and B as well as on Theorem 2.3 stated and proved below, one may guess that there is an absolute constant $c$ such that for every strong semicomplete multipartite digraph $D$, we have $\operatorname{diam}_{\min }(D) \leq \max \{c, \operatorname{diam}(D)\}$. However, the following example shows that this guess is wrong.

To simplify the following discussion, let us first consider the definitions of forward and backward arcs. For an ordering $\alpha=v_{1}, v_{2}, \ldots, v_{n}$ of the vertices of a digraph $D$, an arc $v_{i} v_{j} \in A(D)$ is forward (backward) if $i<j(i>j)$.

For every integer $l \geq 1$, let $D_{l}$ be the semicomplete multipartite digraph with partite sets $V^{\prime}=\left\{x_{0}\right\}, V^{\prime \prime}=\left\{y_{0}\right\}, V_{1}=\left\{x_{1}, y_{1}\right\}, V_{2}=\left\{x_{2}, y_{2}\right\}, \ldots, V_{l}=\left\{x_{l}, y_{l}\right\}$ such that the arcs

$$
x_{0} y_{0}, x_{0} x_{1}, x_{1} x_{2}, \ldots, x_{l-1} x_{l}, y_{0} y_{1}, y_{1} y_{2}, \ldots, y_{l-1} y_{l}
$$

are the only forward $\operatorname{arcs}$ in the ordering $\beta=x_{0}, y_{0}, x_{1}, y_{1}, \ldots, x_{l}, y_{l}$ and $x_{0} y_{0} x_{0}$ is the only cycle of length two in $D_{l}$. 
Observe that the diameter of $D_{l}$ is $l+1$ as $\operatorname{dist}_{D_{l}}\left(x_{0}, y_{l}\right)=\operatorname{dist}_{D_{l}}\left(y_{0}, x_{l}\right)=l+1$ and that the diameter of both $D-x_{0} y_{0}$ and $D-y_{0} x_{0}$ is $l+2$ (while a shortest $\left(x_{0}, y_{l}\right)$-path in $D_{l}$ uses only forward arcs of $\beta$, a shortest $\left(x_{0}, y_{l}\right)$-path in $D_{l}-x_{0} y_{0}$ must use a backward arc). It is not difficult to see that this example can also be modified to semicomplete $k$-partite digraphs with fixed $k \geq 3$ (there will be fewer backward arcs).

In view of this example and Theorems $\mathrm{A}$ and $\mathrm{B}$, we suspect that the following conjecture is correct.

Conjecture 2.1 There is an absolute constant c such that for every strong semicomplete multipartite digraph $D$, we have $\operatorname{diam}_{\min }(D) \leq \operatorname{diam}(D)+c$.

The following two theorems provide further support to this conjecture. Theorem 2.2 shows that the conjecture is true for strong semicomplete multipartite digraphs with a bounded (from above by an absolute constant) number of cycles of length two; Theorem 2.3 indicates that the conjecture is correct for extended semicomplete digraphs. (Theorem 2.3 is also of independent interest as a generalization of Theorem A.)

Theorem 2.2 If $D$ is a strong semicomplete multipartite digraph, $D \neq \overleftrightarrow{K}_{1, n-1}$, containing a cycle of length two, then for every cycle xyx of length two in $D$, we have

$$
\min \{\operatorname{diam}(D-x y), \operatorname{diam}(D-y x)\} \leq \max \{5, \operatorname{diam}(D)+1\} .
$$

Proof: Assume that the theorem is false and that $D$ is a counter-example to the theorem. By Theorem B, we may assume that $D$ is not bipartite. Let $x y x$ be a cycle of length two in $D$ such that

$$
\min \{\operatorname{diam}(D-x y), \operatorname{diam}(D-y x)\}>\max \{5, \operatorname{diam}(D)+1\} .
$$

Therefore, there exist vertices $s_{x y}, t_{x y}, s_{y x}, t_{y x}$ in $D$ such that

$\operatorname{dist}_{D-x y}\left(s_{x y}, t_{x y}\right)>\max \{5, \operatorname{diam}(D)+1\}$ and $\operatorname{dist}_{D-y x}\left(s_{y x}, t_{y x}\right)>\max \{5, \operatorname{diam}(D)+1\}$.

Let $P=p_{0} p_{1} \ldots p_{l}$ be an $\left(s_{x y}, t_{x y}\right)$-path in $D$ of minimum length (in particular, $l \leq$ $\operatorname{diam}(D))$ and let $Q=q_{0} q_{1} \ldots q_{m}$ be an $\left(s_{y x}, t_{y x}\right)$-path in $D$ of minimum length (in particular, $m \leq \operatorname{diam}(D))$. Let $\rho$ and $\eta$ be defined such that $x y=p_{\rho} p_{\rho+1}$ and $y x=q_{\eta} q_{\eta+1}$.

We now consider the following cases, which exhaust all possibilities. Indeed,

$(\rho, \eta) \in[0, l-1] \times[0, m-1]=[0, l-2] \times[0, m-2] \cup[1, l-1] \times[1, m-1] \cup\{(0, m-1)\} \cup\{(l-1,0)\}$, where $[p, q]=\{p, p+1, \ldots, q\}$. 
Case 1: $\rho+1<l, \eta+1<m$. The vertex $p_{\rho+2}$ must belong to the same partite set as $x$, since otherwise $y \rightarrow p_{\rho+2} \rightarrow x$ (by the minimality of $l$ ). This would give us a contradiction against $\operatorname{dist}_{D-y x}\left(s_{y x}, t_{y x}\right)>\max \{5, \operatorname{diam}(D)+1\}$. Analogously, $q_{\eta+2}$ belongs to the same partite set as $y$. Since $p_{\rho+2}$ belongs to the same partite set as $x$ and $q_{\eta+2}$ belongs to the same partite set as $y, p_{\rho+2}$ and $q_{\eta+2}$ are adjacent. If $q_{\eta+2} \rightarrow p_{\rho+2}$, then $\operatorname{dist}_{D-x y}\left(s_{x y}, t_{x y}\right)=\operatorname{dist}_{D}\left(s_{x y}, t_{x y}\right)$, a contradiction. Analogously, if $p_{\rho+2} \rightarrow q_{\eta+2}$, then we also get a contradiction.

Case 2: $\rho>0, \eta>0$. This case can be transformed into Case 1 by considering the converse of $D$.

Case 3: $\rho=0, \eta+1=m$. Since $D$ is not bipartite, there exists a vertex in a partite set different from those of $x$ and $y$. Let $w$ be such a vertex. Clearly, if $x \rightarrow w \rightarrow y$ or $y \rightarrow w \rightarrow x$, then we would get a contradiction. Thus, either $\{x, y\} \rightarrow w$ or $w \rightarrow\{x, y\}$. Assume that $\{x, y\} \rightarrow w$ (the other case can be handled analogously).

Let $R=r_{0} r_{1} \ldots r_{t}$ be a shortest path from $w$ to $p_{l}$ in $D$. Clearly, $R$ must use the arc $x y$, since otherwise there is a path from $x=p_{0}$ to $p_{l}$ of length at most $\operatorname{diam}(D)+1$ in $D-x y$, a contradiction. Let $r_{i}=x$ and observe that $i>1$.

If $\eta=0$, then the path $y r_{0} r_{1} \ldots r_{i}$ is a path from $q_{0}$ to $q_{m}$ of length at most $\operatorname{diam}(D)+1$ in $D-x y$, a contradiction. Therefore $\eta \geq 1$. Either $r_{i}$ or $r_{i-1}$ is adjacent to $q_{\eta-1}$, and clearly the arc must go from $r_{i}$ or $r_{i-1}$ to $q_{\eta-1}$. If $r_{i} \rightarrow q_{\eta-1}$, then we have a path of length 2 from $x$ to $y$, a contradiction, so $r_{i-1} \rightarrow q_{\eta-1}$. However, now the path

$$
x r_{0} r_{1} \ldots r_{i-1} q_{\eta-1} r_{i+1} r_{i+2} \ldots r_{t}
$$

is a $\left(p_{0}, p_{l}\right)$-path of length at most $\operatorname{diam}(D)+1$ in $D-x y$, a contradiction.

Case 4: $\eta=0, \rho+1=l$. This case can be transformed into Case 3 by considering the converse of $D$.

Theorem 2.3 If $T$ is a strong semicomplete digraph with $n$ vertices, $n \geq 3$, and $s_{i} \geq 1$ for $i=1,2, \ldots, n$, then

$$
\operatorname{diam}_{\min }\left(T\left(s_{1}, s_{2}, \ldots s_{n}\right)\right) \leq \max \{3, \operatorname{diam}(T)\}
$$

Proof: By Theorem A, there exists an orientation $T^{\prime}$ of $T$ such that

$$
\operatorname{diam}\left(T^{\prime}\right) \leq \max \{3, \operatorname{diam}(T)\}
$$

Let $D=T\left(s_{1}, s_{2}, \ldots, s_{n}\right)$ and $D^{\prime}=T^{\prime}\left(s_{1}, s_{2}, \ldots, s_{n}\right)$. We will show that $D^{\prime}$ is an orientation of $D$ such that

$$
\operatorname{diam}\left(D^{\prime}\right) \leq \max \left\{3, \operatorname{diam}\left(T^{\prime}\right)\right\} \leq \max \{3, \operatorname{diam}(T)\},
$$


which would complete the proof.

Let $V_{1}, V_{2}, \ldots, V_{n}$ be the partite sets of the semicomplete $n$-partite digraph $D ;\left|V_{i}\right|=s_{i}$ for $i=1,2, \ldots, n$. Let $x$ and $y$ be arbitrary vertices in $D^{\prime}$ and assume that $x \in V_{i}$ and $y \in V_{j}$. If $i \neq j$, then the path from $i$ to $j$ of length at most $\operatorname{diam}\left(T^{\prime}\right)$ in $T^{\prime}$ can be easily transformed into a path from $x$ to $y$ of length at most $\operatorname{diam}\left(T^{\prime}\right)$ in $D^{\prime}$. So, we may assume that $i=j$. Let $V^{\prime}$ be the set of all the vertices dominated by $V_{i}$ in $D^{\prime}$ and $V^{\prime \prime}$ the set of all the vertices dominating $V_{i}$. Clearly, $V\left(D^{\prime}\right)=V_{i} \cup V^{\prime} \cup V^{\prime \prime}$ and there must be an arc $u w$ from $V^{\prime}$ to $V^{\prime \prime}$, as otherwise $T^{\prime}$ would not be strong. Now the path $x u w y$ is a path of length 3 in $D^{\prime}$, so we are done.

The sharpness of the bound of this theorem follows from the sharpness of the bound of Theorem A. Theorem 2.3 also generalizes the following result.

Theorem $\mathbf{C}[5,14]$ If $G$ is a complete $k$-partite (undirected) graph and $k \geq 3$, then $\operatorname{diam}_{\min }(G) \leq 3$, and the bound is sharp.

\section{$3 \quad$ Extended digraphs}

The aim of this section is to provide a generalization to the following theorem as well as to some other results in [12].

Theorem D [12] For a connected graph $G$ of order $n \geq 3$ and integers $s_{1}, s_{2}, \ldots, s_{n} \geq 2$, we have $\operatorname{diam}(G) \leq \operatorname{diam}_{\min }\left(G\left(s_{1}, s_{2}, \ldots, s_{n}\right)\right) \leq \operatorname{diam}(G)+2$.

Theorem $\mathrm{D}$ is valid for $n \geq 3$. The case $n=2$ is covered in the following result.

Theorem $\mathbf{E}[19]$ If $s_{1} \geq s_{2} \geq 2$, then $\operatorname{diam}_{\min }\left(K_{s_{1}, s_{2}}\right)=3$ for $s_{1} \leq\left(\begin{array}{c}s_{2} \\ \left.s_{2} / 2\right\rfloor\end{array}\right)$, and $\operatorname{diam}_{\min }\left(K_{s_{1}, s_{2}}\right)=4$, otherwise.

Theorem 3.1 Let $H$ be a strong digraph of order $n \geq 3$ and let $D=H\left(s_{1}, s_{2}, \ldots, s_{n}\right)$ with $s_{i} \geq 2,1 \leq i \leq n$. Then $\operatorname{diam}(H) \leq \operatorname{diam}_{\min }(D) \leq \operatorname{diam}(H)+2$.

Theorem 3.1 can be proved similarly to Theorem D with only some minor alterations and additions needed. However, we provide a proof of Theorem 3.1 for the sake of completeness.

The requirement $n \geq 3$ is important as one can see from Theorem $\mathrm{E}\left(\operatorname{diam}\left(K_{2}\right)=1\right.$, but $\operatorname{diam}_{\min }\left(K_{s, 2}\right)=4$ for $\left.s \geq 3\right)$. Clearly, $\operatorname{diam}(H) \leq \operatorname{diam}\left(D^{\prime}\right)$ for every orientation $D^{\prime}$ of $D$. To prove the more difficult part of the inequality in Theorem 3.1, we will use the following lemma. 
Lemma 3.2 Let $t_{i}, s_{i}$ be integers such that $2 \leq t_{i} \leq s_{i}$ for $1 \leq i \leq n$ and let $H$ be $a$ strong digraph with vertices $1,2, \ldots, n, n \geq 3$. If the digraph $D^{\prime}=H\left(t_{1}, t_{2}, \ldots, t_{n}\right)$ admits an orientation $F^{\prime}$ in which every vertex $v=(p, i)$, such that $i$ belongs to a cycle in $H$ of length two, lies on a cycle $C_{v}$ of length not exceeding $m$, then $D=H\left(s_{1}, s_{2}, \ldots, s_{n}\right)$ has an orientation $F$ with diameter at most $\max \left\{m, \operatorname{diam}\left(F^{\prime}\right)\right\}$.

Proof: Given an orientation $F^{\prime}$ of $D^{\prime}$, we define an orientation $F$ of $D$ as follows. We have $(p, i) \rightarrow(q, j)$ in $F$ if and only if one of the following holds:

(a) $p<t_{i}, q<t_{j}$ and $(p, i) \rightarrow(q, j)$ in $F^{\prime}$.

(b) $p<t_{i}, q \geq t_{j}$ and $(p, i) \rightarrow\left(t_{j}, j\right)$ in $F^{\prime}$.

(c) $p \geq t_{i}, q<t_{j}$ and $\left(t_{i}, i\right) \rightarrow(q, j)$ in $F^{\prime}$.

(d) $p \geq t_{i}$ and $q \geq t_{j}$ and $\left(t_{i}, i\right) \rightarrow\left(t_{j}, j\right)$ in $F^{\prime}$.

Let $u=(p, i)$ and $v=(q, j)$ be a pair of distinct vertices in $F$. If $i \neq j$, then it is clear that $\operatorname{dist}_{F}(u, v) \leq \operatorname{diam}\left(F^{\prime}\right)$ (we can use obvious modifications of the corresponding paths in $F^{\prime}$ ). We have the same result if $i=j$ but $p<t_{i}$ or $q<t_{j}$. Assume that $i=j, p \geq t_{i}$ and $q \geq t_{j}$. If $i$ belongs to a cycle in $H$ of length two, then using the cycle $C_{u}$ we conclude that $\operatorname{dist}_{F}(u, v) \leq m$. If $i$ belongs to no cycle in $H$ of length two, then since $u, v$ dominate and are dominated by the same vertices and since $\operatorname{dist}_{F}((1, i),(2, i)) \leq \operatorname{diam}(F)$, we have $\operatorname{dist}((p, i),(q, i)) \leq \operatorname{diam}(F)$.

Proof of Theorem 3.1: We prove that there exists an orientation $D^{\prime}$ of $D$ such that $\operatorname{diam}\left(D^{\prime}\right) \leq \operatorname{diam}(H)+2$. If $\operatorname{diam}(H)=1$, then this claim follows from Theorem C. Thus, we may assume that $\operatorname{diam}(H) \geq 2$.

Define an orientation $F^{\prime}$ of $H\left(t_{1}, t_{2}, \ldots, t_{n}\right)$, where every $t_{i}=2$, as follows:

$$
(1, i) \rightarrow(1, j) \rightarrow(2, i) \rightarrow(2, j) \rightarrow(1, i) \text { if and only if } i<j .
$$

Let $u=(p, i)$ and $v=(q, j)$ be a pair of distinct vertices in $F^{\prime}$. We show that $\operatorname{dist}_{F^{\prime}}(u, v) \leq \operatorname{diam}(H)+2$. Suppose that $i k_{1} k_{2} \ldots k_{s} j$ is a path of length $s+1=\operatorname{dist}_{H}(i, j)$ in $H$. Then the path $Q=(p, i)\left(k_{1}^{*}, k_{1}\right)\left(k_{2}^{*}, k_{2}\right) \ldots\left(k_{s}^{*}, k_{s}\right)\left(j^{*}, j\right)$, where $x^{*}=1$ or 2 , is of length $\operatorname{dist}_{H}(i, j)$ in $F^{\prime}$. If $j^{*}=q$, then the last inequality follows. Otherwise, i.e. $j^{*} \neq q$, the path $Q\left(3-k_{s}^{*}, k_{s}\right)(q, j)$ is of length $\operatorname{dist}_{H}(i, j)+2$ in $F^{\prime}$. Thus, $\operatorname{dist}_{F^{\prime}}(u, v) \leq$ $\operatorname{diam}(H)+2$. Hence, $\operatorname{diam}\left(F^{\prime}\right) \leq \operatorname{diam}(H)$. By (1), every vertex $(p, i)$ of $F^{\prime}$, such that $i$ lies on a cycle in $H$ of length two, belongs to a cycle of length 4 . Now this theorem follows from Lemma 3.2.

The following result as well as its corollary can be proved similarly to Theorem 2 and its corollary in [12]. 
Theorem 3.3 Let $H$ be a strong digraph of order $n \geq 3$ and let $D=H\left(s_{1}, s_{2}, \ldots, s_{n}\right)$ with $s_{i} \geq 4,1 \leq i \leq n$ be of diameter at least four. Then $\operatorname{diam}_{\min }(D)=\operatorname{diam}(H)$.

Corollary 3.4 Let $H$ be a strong digraph of order $n \geq 3$ and let $D=H\left(s_{1}, s_{2}, \ldots, s_{n}\right)$ with $s_{i} \geq 4,1 \leq i \leq n$ be of diameter at least three. Then $\operatorname{diam}_{\min }(D) \leq \operatorname{diam}(H)+1$.

Some other results in [12] can also be generalized to orientations of digraphs (the results on cycles). Finally, we would like to suggest the following generalization of the conjecture in [12] (the conjecture in [12] is the same as Conjecture 3.5, but only for connected undirected graphs). The conjecture in [12] seems quite difficult; it was settled for trees in [20].

Conjecture 3.5 Let $H$ be a strong digraph of order $n \geq 3$ and let $D=H\left(s_{1}, s_{2}, \ldots, s_{n}\right)$ with $s_{i} \geq 2,1 \leq i \leq n$ be of diameter at least three. Then $\operatorname{diam}_{\min }(D) \leq \operatorname{diam}(H)+1$.

This conjecture is correct for semicomplete digraphs $H$, see Theorem 2.3 , and $H=\overleftrightarrow{T}$, where $T$ is a tree [20].

\section{Acknowledgements}

Part of this work was done when the first author was visiting the Department of Mathematics, National University of Singapore. The departmental hospitality and financial support are very much appreciated.

\section{References}

[1] J. Bang-Jensen and G. Gutin, Digraphs. Theory, Algorithms and Applications, Springer-Verlag, London, 2000.

[2] F. Boesch and R. Tindell, Robbins's theorem for mixed multigraphs. Amer. Math. Monthly 87 (1980) 716-719.

[3] V. Chvátal and C. Thomassen, Distances in orientations of graphs. J. Combin. Theory Ser. B 24 (1978) 61-75.

[4] F.R.K. Chung, M.R. Garey and R.E. Tarjan, Strongly connected orientations of mixed multigraphs. Networks 15 (1985) 477-484.

[5] G. Gutin, $m$-sources in complete multipartite digraphs, Vestsi Acad. Navuk BSSR Ser. Fiz.-Mat. no. 5 (1989) 101-106 (in Russian).

[6] G. Gutin, Minimizing and maximizing the diameter in orientations of graphs. Graphs and Combinatorics 10 (1994) 225-230.

[7] G. Gutin and A. Yeo, Orientations of digraphs almost preseving diameter, Discrete Applied Math., to appear.

[8] K.M. Koh and E.G. Tay, On optimal orientations of cartesian products of graphs (I). Discrete Math. 190 (1998) 115-136. 
[9] K.M. Koh and E.G. Tay, On optimal orientations of cartesian products of trees. Graphs and Combinatorics, to appear.

[10] K.M. Koh and E.G. Tay, On optimal orientations of cartesian products with a bipartite graph. Discrete Applied Math. 98 (1999) 103-120.

[11] K.M. Koh and E.G. Tay, On optimal orientations of cartesian products of graphs (II): Complete graphs and even cycles. Discrete Math. 211 (2000) 75-102.

[12] K.M. Koh and E.G. Tay, On optimal orientations of $G$ vertex-multiplications. Discrete Math. 219 (2000) 153-171.

[13] J.-C. König, D.W. Krumme and E. Lazard, Diameter preserving orientation of the torus. Networks 32 (1998) $1-11$.

[14] J. Plesník, Remarks on diameters of orientations of graphs. Acta Math. Univ. Comenian. $46 / 47$ (1985) 225236.

[15] F.S. Roberts and Y. Xu, On the optimal strongly connected orientations of city street graphs I: Large grids. SIAM J. Discrete Math. 1 (1988) 199-222.

[16] F.S. Roberts and Y. Xu, On the optimal strongly connected orientations of city street graphs II: Two east-west avenues or north-south streets. Networks 19 (1989) 221-233.

[17] F.S. Roberts and Y. Xu, On the optimal strongly connected orientations of city street graphs III: Three east-west avenues or north-south streets. Networks 22 (1992) 109-143.

[18] F.S. Roberts and Y. Xu, On the optimal strongly connected orientations of city street graphs IV: Four eastwest avenues or north-south streets. Discrete Applied Math. 49 (1994) 331-356.

[19] L. Šoltés, Orientations of graphs minimizing the radius or the diameter. Math. Slovaca 36 (1986) 289-296.

[20] E.G. Tay, Optimal orientations of graphs, PhD thesis, National University of Singapore, 1999.

[21] L. Volkmann, Spanning multipartite tournaments of semicomplete multipartite digraphs. ARS Combinatoria 8 (2001) 271-278. 